\title{
Characteristics of Arrested Seeds in Mukaku Kishu-type Seedless Citrus
}

\author{
Atsu Yamasaki ${ }^{1}$, Akira Kitajima ${ }^{1 *}$, Norihiro Ohara ${ }^{2 * *}$, Mitsutoshi Tanaka ${ }^{2}$ \\ and Kojiro Hasegawa ${ }^{3}$
}

${ }^{1}$ Graduate School of Agriculture, Kyoto University, Hattyonawate, Takatsuki 569-0096, Japan

${ }^{2}$ Kochi Prefectural Agriculture Research Center, Asakura, Kochi 780-8064, Japan

${ }^{3}$ Faculty of Agriculture, Kochi University, Monobe, Nankoku 783-0093, Japan

\begin{abstract}
The expression of seedlessness derived from 'Mukaku Kishu' (Citrus kinokuni hort. ex Tanaka) was investigated histologically. Neither perfect nor imperfect seeds found in seedy cultivars/offspring were formed in 'Mukaku Kishu' and its seedless descendants. Among 'Mukaku Kishu' and its seedless descendants, the fertilized seed type (termed "type A seeds") with an immature, soft seed coat was specifically observed. In most seedless cultivars/ offspring, all embryos of type A seeds were arrested at the zygote or proembryo stage, and embryo development was arrested 10 weeks after pollination. These results indicate that the expression of Mukaku Kishu-type seedlessness is characteristic of a forming type A seed with an immature seed coat and an embryo arrested at an early stage. Endosperm abortion in type A seeds was not observed. Therefore, we demonstrated that an arrested embryo development in the type A seed is not caused by endosperm abortion. Because the expression of Mukaku Kishu-type seedlessness is not controlled by the embryo genotype, type A seed production would not be caused by gene expression in embryos but by that in maternal tissues, more likely in the immature seed coat tissue.
\end{abstract}

Key Words: embryo development, endosperm development, histological study, seed coat, seed formation.

\section{Introduction}

Citrus is one of the most economically important fruits in the world, and the production of seedless cultivars is very desirable for both growers and consumers. However, genetic variation in the seed number per fruit in citrus is high, ranging from seedlessness, e.g., 'Okitsuwase' satsuma mandarin (Citrus unshiu Marc.), to very seedy, e.g., a mean of 62 seeds/fruit in 'Hirado Buntan' pummelo [C. maxima (Burm.) Merr.] (Yamamoto et al., 1992).

Kishu (C. kinokuni), which was cultivated widely for several hundred years until the nineteenth century in Japan (Hodgson, 1967; Tanaka, 1954), is seedy, such as 'Hira Kishu', excluding seedless 'Mukaku Kishu'. The seedless 'Mukaku Kishu', which is diploid $(2 \mathrm{n}=18)$ (Befu et al., 2002), is speculated to be a bud mutant of the seedy Kishu. 'Mukaku Kishu' produces completely

Received; February 22, 2008. Accepted; June 24, 2008.

A part of this work was presented in 2004 at the Autumn Meeting of the Japanese Society for Horticultural Science.

* Corresponding author (E-mail: kitajima@kais.kyoto-u.ac.jp).

** Present address: Kochi Prefectural Central West Agriculture Promotion Center, Takaoka, Tosa 781-1102, Japan. seedless fruits even on pollination by hand (Iwamasa, 1978; Nagai and Tanikawa, 1928; Nesumi et al., 1992). Since the seedless characteristic of 'Mukaku Kishu' is inheritable (Nesumi et al., 2001), 'Mukaku Kishu' has attracted considerable attention as a breeding parent to produce seedless cultivars. A newly released seedless cultivar is 'Southern Yellow', which originated from a cross between 'Tanikawa Buntan' pummelo (an unidentified hybrid with C.maxima) and 'Mukaku Kishu' (Kobayashi, 1995; Yoshida et al., 2005a, 2005b).

In a previous study (Yamasaki et al., 2007), it was indicated that the expression of seedlessness in 'Mukaku Kishu' and its descendants was not derived from abnormal embryo sacs and unfertilized ovules, but from arrested seed development at an early stage after fertilization. However, the stages at which embryo development is arrested and the timing following fertilization in 'Mukaku Kishu' and its seedless descendants are still unclear.

Nesumi et al. (2001) proposed that the seedless characteristics derived from 'Mukaku Kishu' were controlled by a dominant gene $(F s)$ and a repressor gene (Is) that dominantly inhibits seedlessness expression by Fs. To identify these genes, it is necessary to characterize the expression of the seedlessness phenotype derived 
from 'Mukaku Kishu'. Therefore, the objective of this study was to investigate the morphological processes leading to arrested embryo development and to characterize the expression of seedlessness in 'Mukaku Kishu' and its descendants in comparison with embryo development in seedy cultivars/offspring.

\section{Materials and Methods}

\section{Plant materials}

'Hira Kishu', 'Mukaku Kishu', 'Southern Yellow', Bu1-7 pummelo, and five plants (BSY 1, BSY 3, BSY 10, BSY 12, and BSY 18) of the BSY were used (Fig. 1). Bu1-7 pummelo is an open pollinated seedling of 'Suisho Buntan' pummelo (C. maxima). The BSY offspring were derived from a cross between Bu1-7 pummelo and 'Southern Yellow' in 1994 and the grafting of seedlings onto 34-year-old 'Nankan No. 4' satsuma mandarin (C. unshiu Marc.) interstock in 1995. All plants were grown at the Fruit Tree Research Institute of Kochi Prefectural Agriculture Research Center, Kochi, Japan in 2003.

Flowers of the cultivars/offspring used in this study were hand-pollinated with Hyuganatsu (C.tamurana hort. ex Tanaka) pollen at anthesis. Five fruits per cultivar/offspring were collected $6,8,10,12,14,16,18$, and 26 weeks after pollination (WAP), and seeds were extracted.

\section{Measurement of seed growth}

In all cultivars except the BSY offspring, the longitudinal length of seeds was measured under a stereoscopic microscope during 6 to $26 \mathrm{WAP}$. The seed types were grouped visually into perfect (normally developing), imperfect (poorly developing), and ovulelike (unfertilized ovules and very small-sized) seeds from 10 to 26 WAP. The seeds at 6 and 8 WAP were not grouped because of their similar size.

\section{Histological observation}

To investigate the development of embryo and endosperm tissues, seeds were fixed in FAA (formalin: glacial acetic acid: 70\% ethanol, $5: 5: 90 \mathrm{v} / \mathrm{v} / \mathrm{v}$ ), and, subsequently, a clearing-staining procedure was performed, as described previously (Yamasaki et al., 2007). The outer and inner integuments of cleared seeds were removed by tweezers or fine needles under a stereoscopic microscope. The seeds were mounted in BB-4 1/2 fluid on a microscope slide, and examined with a microscope (BX50, Olympus, Tokyo, Japan) equipped with differential interference contrast optics. The samples in which the interior of an embryo sac, such as the zygote or embryo, could not be discerned were treated as exhibiting embryo sac degeneration. Since embryo sac degeneration was observed in all cultivars/offspring, these samples were regarded as unfertilized ovules.

\section{Fruit characteristics at harvest}

Ten hand- and open-pollinated fruits were harvested on 24 December and average number of seeds per fruit was measured. Seeds were categorized visually into the three types described above.

\section{Results}

\section{Seed formation}

In all seedy cultivars and BSY offspring except BSY 10 and BSY 18 , the percentages of perfect and imperfect seeds per fruit were 45.9 to $76.2 \%$, confirming that these cultivars/offspring are seedy (Table 1). On the other hand, neither perfect nor imperfect seeds similar to seedy cultivars/offspring were found in 'Mukaku Kishu', 'Southern Yellow', BSY 10, and BSY 18. These cultivars/offspring were thus practically seedless, although they had very small seeds, which were observed visually to have an immature and soft seed coat, and swollen appearance (Fig. 2). Although the size of this seed type was similar to that of ovulelike seeds from seedy cultivars, there was a typical morphological

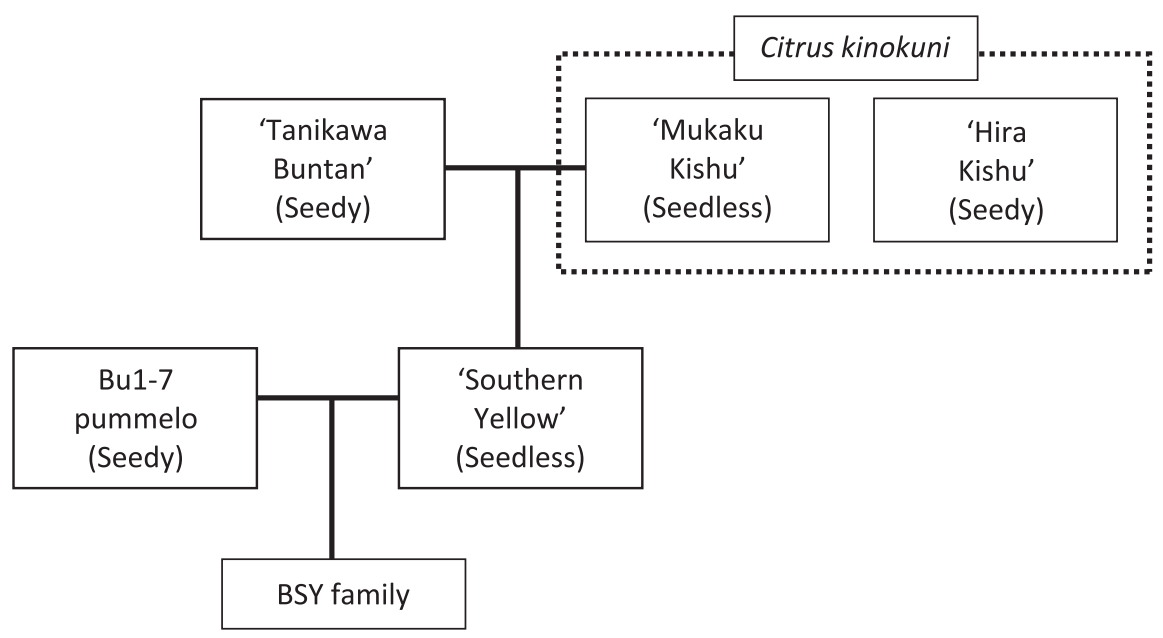

Fig. 1. Schematic family tree of the cultivars/offspring used in this study. 
Table 1. Seed number in BSY offspring, their parents, and grandparents at harvest.

\begin{tabular}{|c|c|c|c|c|c|}
\hline & \multicolumn{4}{|c|}{ Seeds per fruit (no.) } & \multirow{2}{*}{$\begin{array}{c}\text { Perfect and imperfect } \\
\text { seeds }(\%)^{z}\end{array}$} \\
\hline & Perfect seeds & Imperfect seeds & Ovulelike seeds & Type A seeds & \\
\hline 'Hira Kishu’' & 18.0 & 4.5 & 13.2 & 0.0 & 47.9 \\
\hline ‘Mukaku Kishu’ & 0.0 & 0.0 & 9.6 & 9.1 & 0.0 \\
\hline 'Southern Yellow' & 0.0 & 0.0 & 22.2 & 32.8 & 0.0 \\
\hline Bu1-7 pummelo & 10.6 & 5.0 & 12.0 & 0.0 & 58.2 \\
\hline BSY1 & 24.7 & 6.1 & 36.6 & 0.0 & 45.9 \\
\hline BSY3 & 29.8 & 8.5 & 43.8 & 0.0 & 47.3 \\
\hline BSY10 & 0.0 & 0.0 & 32.5 & 31.5 & 0.0 \\
\hline BSY12 & 49.5 & 12.5 & 19.7 & 0.0 & 76.2 \\
\hline BSY18 & 0.0 & 0.0 & 13.0 & 45.0 & 0.0 \\
\hline
\end{tabular}

${ }^{z}$ (Number of perfect and imperfect seeds/total seed number) $\times 100$.

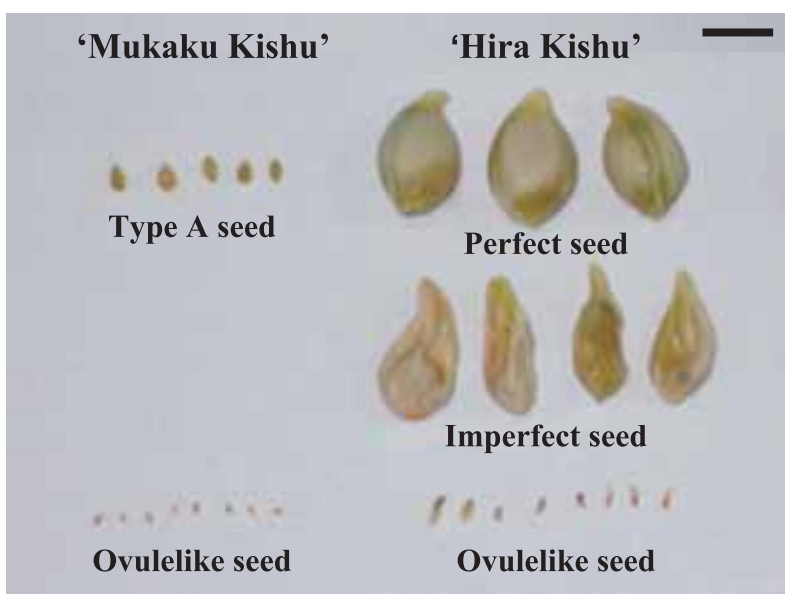

Fig. 2. Comparison of seeds in the seedless 'Mukaku Kishu' and seedy 'Hira Kishu'. Bar $=0.5 \mathrm{~cm}$.
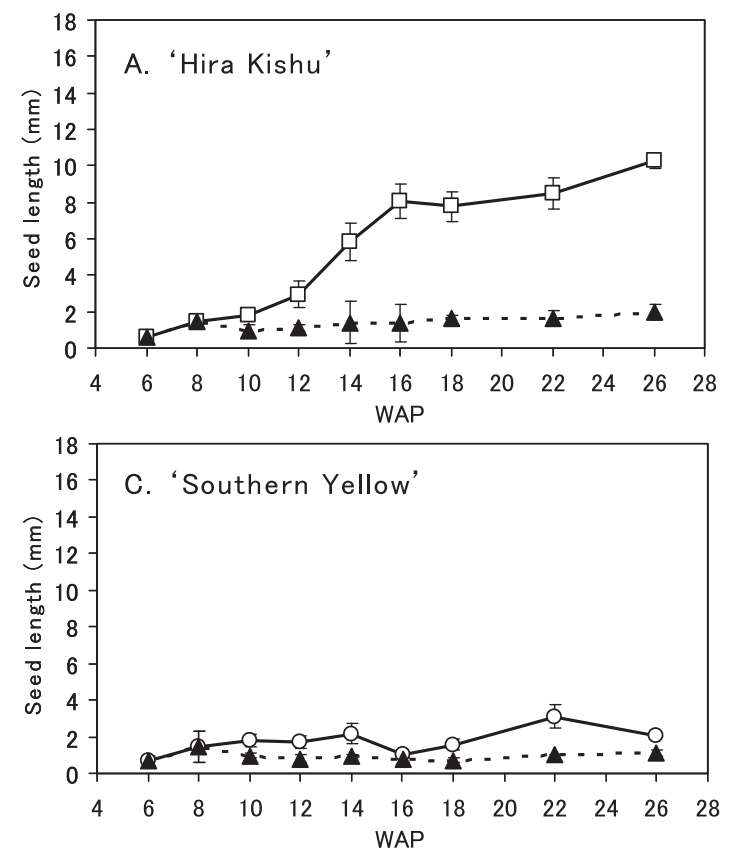

difference between this type of swollen seed and the squashed ovulelike seeds. The swollen seeds were termed "type A seeds". Type A seeds were specific to 'Mukaku Kishu' and its seedless descendants, and were not observed in seedy cultivars/offspring.

\section{Seed development}

In seedless cultivars, the slight growth of type A seeds was observed. Seeds were 1-2 mm long at $10 \mathrm{WAP}$, and 2-3 mm long at 26 WAP (Fig. 3B, C). The length of ovulelike seeds was consistently about $1.0 \mathrm{~mm}$ throughout the sampling period. In contrast, in seedy cultivars, perfect seeds grew rapidly from 10 WAP (Fig. 3A, D). At 26 WAP, the length of perfect seeds in 'Hira Kishu' and Bu1-7 pummelo was 10.3 and $13.0 \mathrm{~mm}$, respectively. Although the length of ovulelike seeds in 'Hira Kishu'
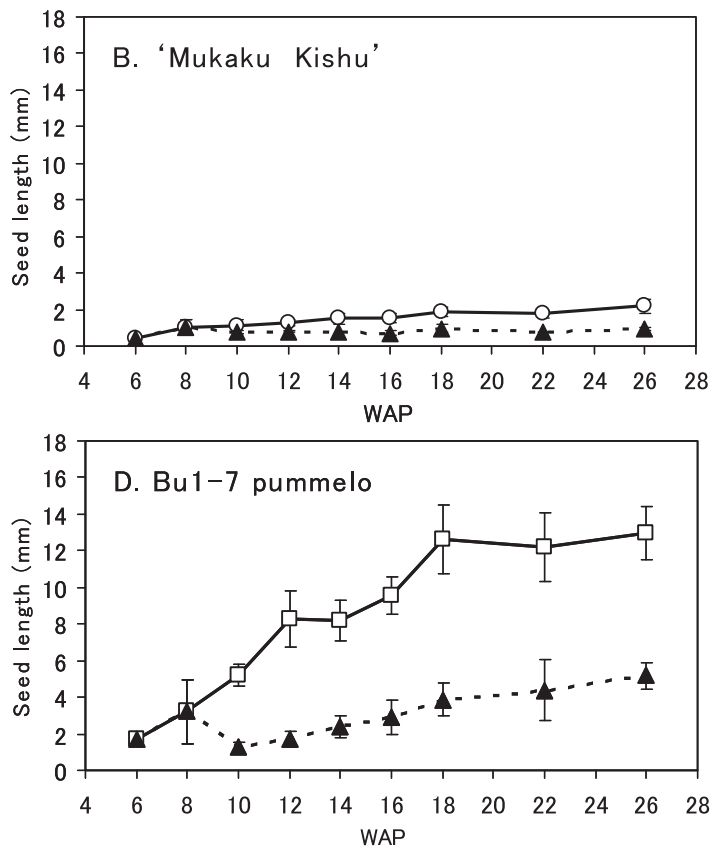

Fig. 3. Longitudinal growth of seeds in seedy 'Hira Kishu' (A), seedless 'Mukaku Kishu' (B), seedless 'Southern Yellow' (C), and seedy Bu17 pummelo (D). WAP, weeks after pollination. $(\square)$ Perfect seeds, $(\boldsymbol{\Delta})$ Ovulelike seeds, $(\bigcirc)$ Type A seeds. Error bars represent the standard deviation of the mean $(n=20)$. 
was less than $2.0 \mathrm{~mm}$, that in Bu1-7 pummelo was up to $5.0 \mathrm{~mm}$.

\section{Embryo development}

At 6 WAP, the embryos in fertilized seeds of two seedless cultivars were still at the zygote stage of development. However, some of the embryos of seedy BSY 1 and BSY 12 had developed to the proembryo stage, while those of the other seedy cultivars/offspring were still at the zygote stage (Fig. 4A).

At $8 \mathrm{WAP}$, embryos at the zygote or proembryo stages were observed in four cultivars/offspring (Fig. 4B). The embryo development of Bu1-7 pummelo was very rapid with $78.6 \%$ of the seeds having reached the globular stage. In seedless cultivars/offspring, 9.1 and 1.6\% of seeds had reached the proembryo stage in 'Southern Yellow' and BSY 18, respectively, while embryos were observed at the zygote stage in 'Mukaku Kishu' and BSY 10.

At 10 WAP, embryos in perfect seeds of two seedy cultivar/offspring excluding 'Hira Kishu' were at the globular or heart-shaped stages, and some BSY 1 embryos were also at the torpedo- or cotyledon-shaped stages (Fig. 4C). In contrast, embryos of type A seeds in the seedless 'Mukaku Kishu' and 'Southern Yellow', and perfect seeds of the seedy 'Hira Kishu', were at the zygote or proembryo stages. Most ovulelike seeds may be identified as unfertilized ovules exhibiting embryo sac degeneration, but, in some cultivars, 5-8\% of the ovulelike seeds were fertilized because a zygote was

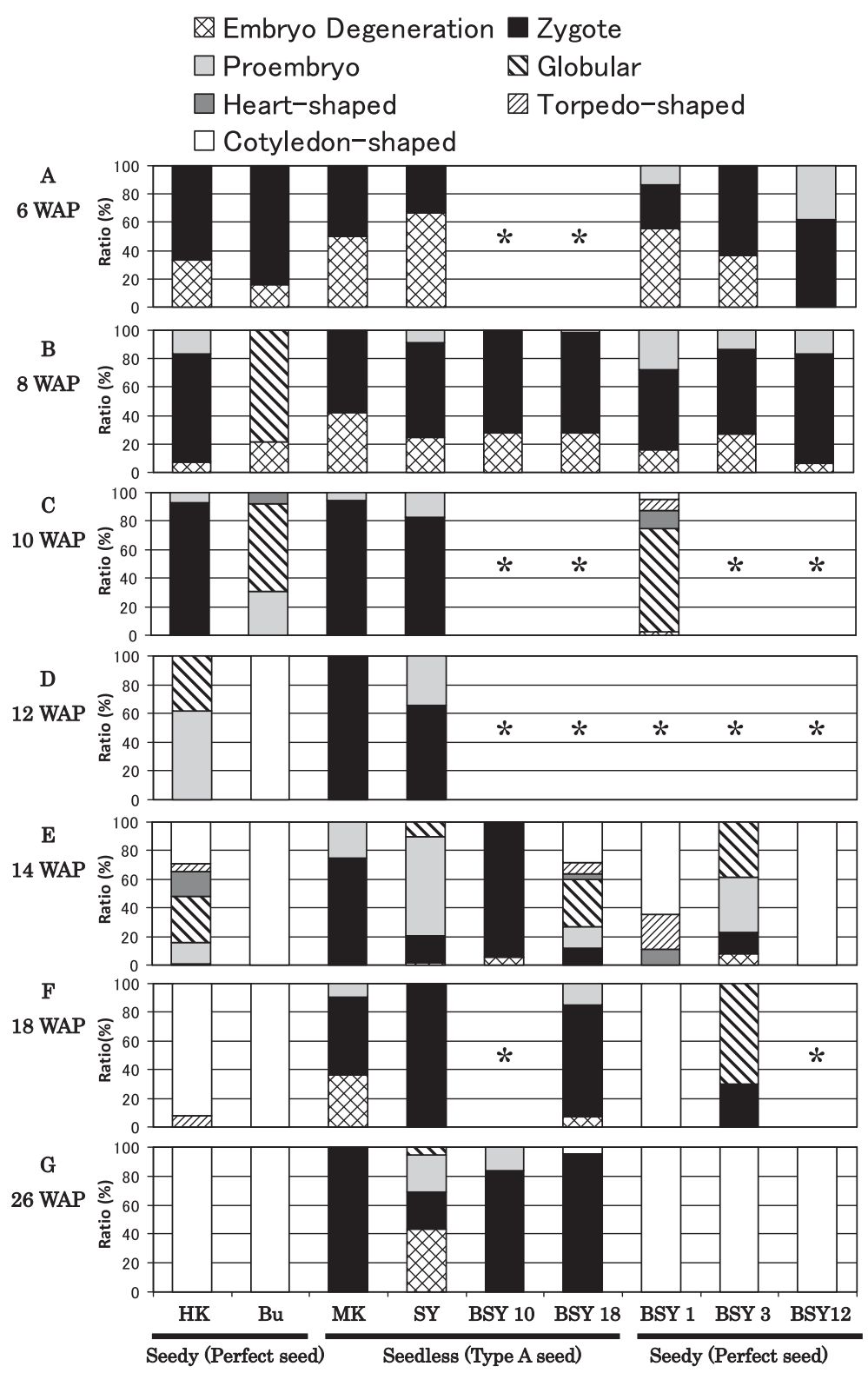

Fig. 4. Percentage of seeds at different embryo developmental stages between 6 and 26 weeks after pollination (WAP) in BSY offspring, their parents, and grandparents. $\mathrm{HK}=$ 'Hira Kishu', $\mathrm{Bu}=\mathrm{Bu}$ 1-7 pummelo, $\mathrm{MK}=$ 'Mukaku Kishu', and $\mathrm{SY}=$ 'Southern Yellow'. Asterisk indicates not examined. 
discernible (data not shown).

At 12 WAP, all perfect seeds in the seedy Bu1-7 pummelo contained embryos at the cotyledon-shaped stage (Fig. 4D). In 'Hira Kishu', embryos had slightly developed compared to $10 \mathrm{WAP}$, and the percentage of embryos at the proembryo and globular stages was 61.5 and $38.5 \%$, respectively. In contrast, type A seeds of the seedless 'Mukaku Kishu' and 'Southern Yellow' remained at the zygote or proembryo stages.

At 14 WAP, in seedy cultivars/offspring, perfect seeds with a mature seed coat were observed. Perfect seeds contained embryos at the cotyledon-shaped stage in four seedy cultivars/offspring including 'Hira Kishu', although the embryos in BSY 3 were at the proembryo or globular stages at most and showed slow development (Fig. 4E). In contrast, embryos of type A seeds in the seedless 'Mukaku Kishu' were arrested at the zygote or proembryo stage. In 'Southern Yellow', however, 10.5\% of the type A seeds had embryos at the globular stage, the embryos in $69.1 \%$ of these seeds remained at the proembryo stage. Almost all type A seeds in the seedless BSY 10 were at the zygote stage (94.1\%), whereas the embryos of BSY 18 ranged from the zygote to cotyledonshaped stages, which differed from the other seedless cultivars/offspring. In seedless BSY 18, seedy BSY 12, and 'Hira Kishu', the length of seeds with a cotyledonshaped embryo was 5.1, 7.9, and $5.7 \mathrm{~mm}$, respectively, and the length of the cotyledon-shaped embryo was 1.3, 2.4 , and $1.6 \mathrm{~mm}$, respectively (data not shown). Although the seed and embryo lengths of BSY 18 were similar to those of 'Hira Kishu', the seed coat of BSY 18 was still soft.

At 18 WAP, in three seedy cultivars/offspring excluding BSY 3 , the embryos in over $70 \%$ of the perfect seeds were at the cotyledon-shaped stage, while the embryos of seedy BSY 3 were at the zygote or globular stages (Fig. 4F). In seedless cultivars/offspring, embryos in type A seeds were at the zygote or proembryo stages, similar to embryo development at 16 WAP (data not shown).

At 26 WAP, in all seedy cultivars/offspring, only cotyledon-shaped embryos were observed in all perfect seeds (Fig. 4G). On the other hand, type A seeds of two seedless cultivars/offspring, 'Mukaku Kishu' and BSY 10 , remained at the zygote or proembryo stages without showing any symptoms of embryo sac degeneration and browning of the inner seed coat. The embryos of 'Southern Yellow' were at the zygote to globular stages despite the observation of slight browning. In seedless BSY 18, seedy BSY 12, and 'Hira Kishu', the length of seeds with a cotyledon-shaped embryo was 7.0, 13.2, and $10.3 \mathrm{~mm}$, respectively, and the length of the cotyledon shaped embryo was $3.8,8.5$, and $7.8 \mathrm{~mm}$, respectively (data not shown). These results indicate that the embryos at the cotyledon-shaped stage in seedy cultivars/offspring grow rapidly, while the embryos in seedless BSY 18 show only marginal growth.

\section{Endosperm development}

Numerous free endosperm nuclei were observed in seeds of 'Hira Kishu' at 10 and 12 WAP (Fig. 5D). However, as embryo development proceeded beyond 14 WAP, no endosperm tissues were observed (Fig. 5E, F). In contrast, several free endosperm nuclei and thin membrane tissue in the embryo sac were observed in type A seeds of 'Mukaku Kishu' at 12 WAP (Fig. 5A), similar to those observed at 3 WAP (data not shown). Even beyond 14 WAP, several free endosperm nuclei and thin membrane tissue in the embryo sac were observed without internal embryo sac degeneration (Fig. 5B). Moreover, at $26 \mathrm{WAP}$, free endosperm nuclei were also observed (Fig. 5C).
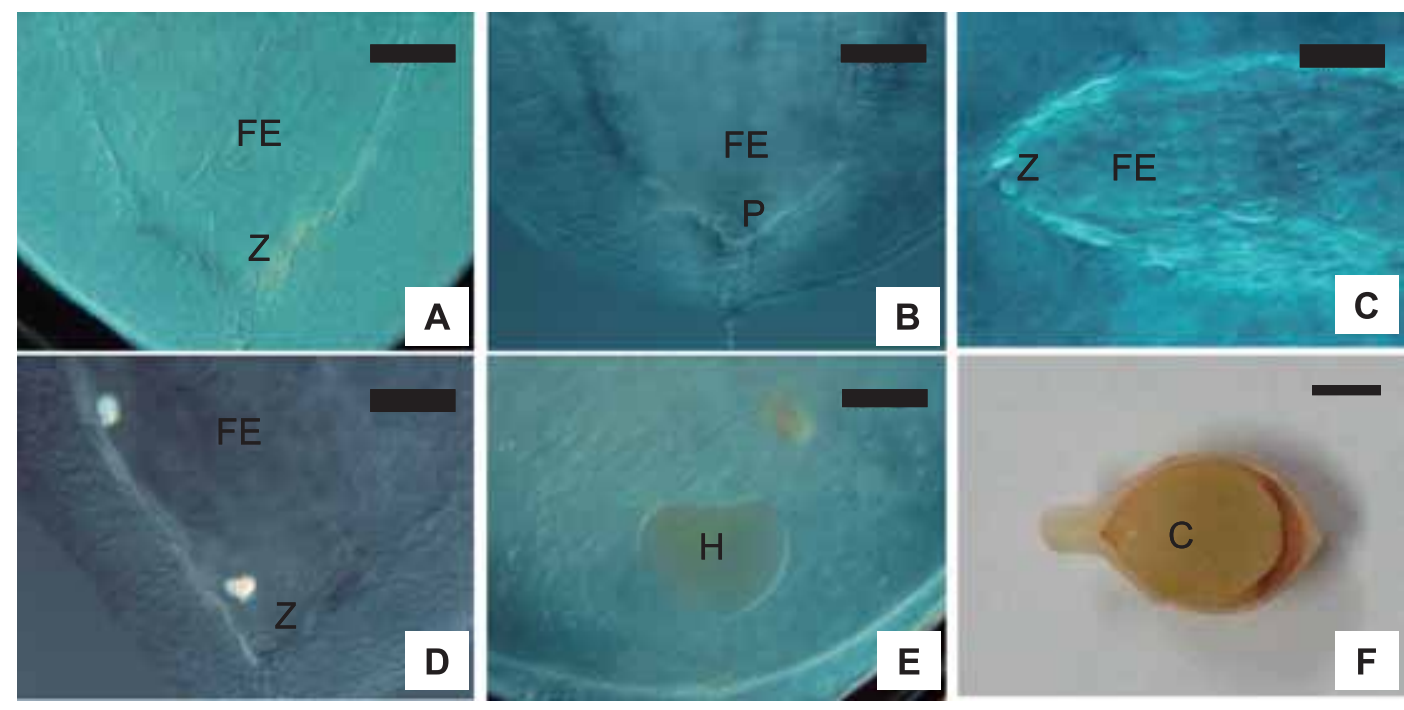

Fig. 5. Free endosperm cells in seedless 'Mukaku Kishu' (A, B, C) and seedy 'Hira Kishu' (D, E, F) at 12 (A, D), 14 (B, E), and 26 (C, F) weeks after pollination. Scale bars $=60 \mu \mathrm{m}(\mathrm{A}-\mathrm{D}), 120 \mu \mathrm{m}(\mathrm{E}), 2.5 \mathrm{~mm}(\mathrm{~F}) ; \mathrm{C}=$ cotyledon-shaped embryo, $\mathrm{FE}=$ free endosperm, $\mathrm{H}=$ heartshaped embryo, $\mathrm{P}=$ proembryo, $\mathrm{Z}=$ zygote. 


\section{Discussion}

In citrus, seedlessness is a desirable trait for fresh fruit consumption and juice processing, and, therefore, this is one of the most important breeding objectives. The phenomena of female sterility and early arrested seed development were demonstrated in certain seedless cultivars/offspring. Although 'Mukaku Kishu' has fully developed embryo sacs (Furusato, 1958), it produces completely seedless fruits even if cross-pollinated (Iwamasa, 1978; Nagai and Tanikawa, 1928; Nesumi et al., 1992). Since this trait is qualitatively inherited, 'Mukaku Kishu' is useful for cross-breeding as a pollen parent (Iwamasa, 1966; Nagai and Tanikawa, 1928; Nesumi et al., 2001).

In a previous study, factors involved in seedless expression were investigated histologically using several cultivars/offspring included in the present study (Yamasaki et al., 2007). Moreover, neither perfect nor imperfect seeds were found in the seedless 'Mukaku Kishu', 'Southern Yellow', BSY 18, and BSY 21. In the present study, we visually observed the seed type that had an immature, soft, and edible seed coat in 'Mukaku Kishu' and its seedless descendants. These type A seeds were clearly different from perfect and imperfect seeds of seedy cultivars/offspring that had a mature, hard, and dried seed coat. Although unfertilized ovules and aborted seeds just after fertilization were previously categorized as ovulelike seeds (Yamasaki et al., 2007), we distinguished these seeds from 10 WAP in this study. Type A seeds were apparently specific to 'Mukaku Kishu' and its seedless descendants. Between 6 and 26 WAP, type A seeds developed only slightly and were a little larger than ovulelike seeds from 10 WAP. Other than in 'Mukaku Kishu' and its seedless descendants, no seeds similar to type A have been observed in citrus. These observations indicate that the expression of seedlessness in these cultivars/offspring is involved in the presence of type A seeds, and is defined as "Mukaku Kishu-type seedlessness".

In addition, it was previously observed that no difference existed in the rates of abnormal embryo sac formation and fertilization between seedless and seedy cultivars/offspring (Yamasaki et al., 2007). A difference between these factors was only apparent in the embryo development stages. Only zygote or early stages of embryo development were present in seedless cultivars/ offspring at $14 \mathrm{WAP}$, whereas embryos at the cotyledonshaped stage existed in seedy cultivars. Therefore, the expression of seedlessness derived from 'Mukaku Kishu' may be caused by a factor associated with the arrest of embryo development. In the present experiments, most of the ovulelike seeds were caused by embryo sac degeneration due to non-fertilization, which was observed from 10 WAP. All type A seeds in seedless cultivars/offspring were fertilized, and embryos at the zygote or proembryo stages were observed at 8 WAP.
However, the embryos remained at these developmental stages beyond 14 WAP. The expression of seedlessness in BSY 10, for which the present experiments represent the first examination of this offspring type, also coincided with that in 'Mukaku Kishu'. In contrast, in seedy cultivars/offspring, embryos at the proembryo stage were observed at $6 \mathrm{WAP}$, the globular stage from 10 to $12 \mathrm{WAP}$, and the cotyledon-shaped stage at 14 WAP. These findings indicate that embryo development in the type A seed was inhibited mainly at the zygote or proembryo stages. We conclude that the expression of Mukaku Kishu-type seedlessness is related to arrested embryo development at an early stage, and that the embryos seem to be arrested after 10 WAP.

Expression patterns of seedlessness have been extensively studied in grape (Vitis vinifera). Two different types of seedlessness have been observed among grape genetic resources: parthenocarpy and stenospermocarpy (Stout, 1936). Stenospermocarpy leads to another form of seedlessness in which the fruit contains partially formed seeds that abort after fertilization. Person (1932) reported that integument development in stenospermic ovules was abnormal and independent of embryo sac development. In addition, $V$. vinifera cv. 'Thompson Seedless' fruits often contain small rudimentary seeds composed of an embryo, arrested at the globular to heart stages, and covered by a soft, edible seed coat (Hanania et al., 2007). In the present study, we identified a similar seed type among 'Mukaku Kishu' and its seedless descendants. These observations indicate that the expression of Mukaku Kishu-type seedlessness might be due to stenospermocarpy similar to that in 'Thompson Seedless', which agrees with the results of Ishii et al. (1999).

Successful development of the embryo depends on normal endosperm development in almost all species (Brink and Cooper, 1947). In citrus, it has been reported that embryo abortion at an early stage is related to imbalance of the chromosome number between the embryo and endosperm (Esen and Soost, 1973). Our results suggested that arrested seed development at an early stage in Mukaku Kishu-type seedlessness is not a result of endosperm abortion, since no endosperm tissues degenerated or disappeared even at 26 WAP.

Nesumi et al. (2001) reported that the ratios of seedless to seedy offspring were $1: 1$ and $1: 3$ in the progenies of 'Mukaku Kishu' obtained by crossing with seedy mandarins and pummelos, respectively. Moreover, these authors proposed that the seedless characteristics derived from 'Mukaku Kishu' might be regulated by two genes $(F s$ and $I s)$. Since all fruits on a tree which have the genotype $F_{s} F_{s}$-isis or $F_{s} f s$-isis are seedless, defective zygote development should not be controlled by the embryo genotype. Although in seedless BSY 18, the cotyledon-shaped embryo was observed at 14 and 26 WAP, the seeds with a cotyledon-shaped embryo were all of type A. The results suggest that some factors to 
promote embryo development may exist. No cotyledonshaped embryo was observed at 18 WAP. This may have been due to the limited number of sampled fruits and small number of developing embryos. These results indicate that the expression of Mukaku Kishu-type seedlessness is characterized by forming type A seeds and arrested embryo development at early stages. Although a cause-and-effect relationship between them remains unclear, the expression of Mukaku Kishu-type seedlessness would be correlated closely with gene expression in maternal tissues, such as the seed coat or nucellus, based on the following results: (1) all fertilized seeds in the fruit are of type A and arrested in early stages of development; (2) seedlessness derived from 'Mukaku Kishu' is inherited by its progenies; and (3) endosperm abortion is absent. Therefore, the arrested embryo development cannot be the primary cause of the expression of Mukaku Kishu-type seedlessness, although it is closely related to seedlessness expression. Incomplete development of the seed coat would be a more likely primary cause of seedlessness expression.

\section{Literature Cited}

Befu, M., A. Kitajima and K. Hasegawa. 2002. Classification of the citrus chromosomes with same types of chromomycin A banding patterns. J. Japan. Soc. Hort. Sci. 71: 394-400 (In Japanese with English abstract).

Brink, R. A. and D. C. Cooper. 1947. The endosperm in seed development. Bot. Rev. 13: 423-541.

Esen, A. and R. K. Soost. 1973. Seed development in citrus with special reference to $2 \mathrm{X} \times 4 \mathrm{X}$ crosses. Amer. J. Bot. 60: 448462.

Furusato, K. 1958. Kankitsu no iden to ikushu 8. Kankitsu. 12: 44-47 (In Japanese).

Hanania, U., M. Velcheva, E. Or, M. Flaishman, N. Sahar and A. Perl. 2007. Silencing of chaperonin 21 , that was differentially expressed in inflorescence of seedless and seeded grapes, promoted seed abortion in tobacco and tomato fruits. Transgenic Res. 16: 515-525.

Hodgson, R. W. 1967. Horticultural varieties of citrus. p. 431591. In: W. Reuther, H. J. Webber and L. D. Batchelor (eds.). The Citrus Industry, vol. 1, History, world distribution, botany and varieties. University of California Press, Berkeley.
Ishii, K., H. Nesumi, T. Takagi and T. Hirabayashi. 1999. Histological observation on seedlessness in 'Mukaku-Kishu' (C. kinokuni hort. ex Tanaka). J. Japan. Soc. Hort. Sci. 68 (Suppl. 2): 173 (In Japanese).

Iwamasa, M. 1966. Studies on the sterility in genus citrus with special reference to the seedlessness. Bul. Hort. Res. Sta. B 6: $1-81$.

Iwamasa, M. 1978. Kankitsu no ikushu nikansuru shomondai 19. Agr. Hort. 53: 105-108 (In Japanese).

Kobayashi, S. 1995. Sinhinshu no saibaigizyutsu. Kazitsu Nihon 50: 101 (In Japanese).

Nagai, K. and T. Tanikawa. 1928. On citrus pollination. Proc. 3rd Pan-Pacific Sci. Congr. Tokyo. 2: 2023-2029.

Nesumi, H., Y. Ito, T. Yoshioka and T. Yoshida. 1992. Female sterility of 'Mukakukishu' and its inheritance. J. Japan. Soc. Hort. Sci. 61 (Suppl. 1): 36-37 (In Japanese).

Nesumi, H., M. Nakano and T. Yoshida. 2001. Mode of inheritance on the abnormal development of impregnated ovules derived from Mukaku-kishu. J. Japan. Soc. Hort. Sci. 70 (Suppl. 2): 403 (In Japanese).

Pearson, H. M. 1932. Parthenocarpy and seedlessness in Vitis vinifera. Science 76: 594.

Stout, A. B. 1936. Seedlessness in grapes. N. Y. State Agr. Expt. Sta. Tech. Bul. 238.

Tanaka, T. 1954. List of species of citrus at the present stage of the investigation. p. 136. In: T. Tanaka (ed.). Species problem in citrus. Japanese Society for the Promotion of Science, Tokyo.

Yamasaki, A., A. Kitajima, N. Ohara, M. Tanaka and K. Hasegawa. 2007. Histological study on expression of seedlessness in Citrus kinokuni 'Mukaku Kishu' and its progenies. J. Amer. Soc. Hort. Sci. 132: 869-875.

Yamamoto, M., Y. Yamada, R. Matsumoto, H. Ikemiya and N. Okudai. 1992. Inheritance of seed number in citrus. Bul. Fruit Tree Res. Sta. 23: 47-56 (In Japanese with English abstract).

Yoshida, T., H. Nesumi, T. Yoshioka, Y. Ito, I. Ueno and Y. Yamada. 2005a. 'Kankitsu Chukanbohon Nou 5 Gou' ('Citrus Parental Line Norin No. 5') is useful for breeding seedless and early maturing cultivars. Bul. Natl. Inst. Fruit Tree Sci. 4: 47-52 (In Japanese with English abstract).

Yoshida, T., H. Nesumi, T. Yoshioka, Y. Ito, M. Yano, M. Nakano, I. Ueno, Y. Yamada, K. Ogawa, S. Murase, F. Takishita, T. Hidaka and S. Kawai. 2005b. 'Kankitsu Chukanbohon Nou 6 Gou' ('Citrus Parental Line Norin No. 6') is useful for breeding seedless and functional component-rich cultivars. Bul. Natl. Inst. Fruit Tree Sci. 4: 53-59 (In Japanese with English abstract). 